\title{
Cancer Treatment Research Foundation
}

National Cancer Institute

\section{Source}

National Cancer Institute. Cancer Treatment Research Foundation. NCI Thesaurus. Code C39349.

The Cancer Treatment Research Foundation (CTRF) is a non-profit organization committed to the eradication of cancer by providing the most innovative and promising patient-driven clinical research that delivers immediate treatment options. CT RF stimulates and funds research relevant to cancer therapy (e.g., new agents, immunotherapy, biological response modifiers, gene therapy), clinical nutrition, quality of life and cancer integrative medicine. 